PSICOLOGÍA

IBEROAMERICANA
Psicología Iberoamericana ISSN: 1405-0943

revista.psicologia@ibero.mx

Universidad Iberoamericana, Ciudad de México México

\title{
La teoría de la sensibilidad al reforzador como predictor de psicopatía y conducta delincuencial: datos obtenidos en estudiantes universitarios
}

Pulido Rull, Marco Antonio; Flores Herrera, Alexa; Mendoza Montes, Pamela

La teoría de la sensibilidad al reforzador como predictor de psicopatía y conducta delincuencial: datos obtenidos en estudiantes universitarios

Psicología Iberoamericana, vol. 29, núm. 2, e292397, 2021

Universidad Iberoamericana, Ciudad de México, México

Disponible en: https://www.redalyc.org/articulo.oa?id=133967822004

DOI: https://doi.org/10.48102/pi.v29i2.397

Los autores/as conservan los derechos de autor y ceden a la revista el derecho de la primera publicación, con la obra registrada bajo la Licencia Creative Commons Atribución 4.0 Internacional.

Los autores/as conservan los derechos de autor y ceden a la revista el derecho de la primera publicación, con la obra registrada bajo la Licencia Creative Commons Atribución 4.0 Internacional.

\section{(c) (7)}

Esta obra está bajo una Licencia Creative Commons Atribución 4.0 Internacional. 
Artículos

\section{La teoría de la sensibilidad al reforzador como predictor de psicopatía y conducta delincuencial: datos obtenidos en estudiantes universitarios}

Reinforcement sensitivity theory as a predictor of psychopathy and delinquent behavior: Data obtained from samples of college students

Marco Antonio Pulido Rull mpulidor100@gmail.com Universidad Intercontinental y Centro de Estudios Superiores Monte

Psicología Iberoamericana, vol. 29, núm. 2, e292397, 2021

Universidad Iberoamericana, Ciudad de México, México

Recepción: 27 Mayo 2021

Aprobación: 06 Julio 2021

DOI: https://doi.org/10.48102/ pi.v29i2.397

Redalyc: https://www.redalyc.org/ articulo.oa?id=133967822004 Fénix, México

Fénix, México
(iD) https://orcid.org/0000-0002-0800-962X
Alexa Flores Herrera alexaherrera259@gmail.com
Universidad Anáhuac, México
(D) https://orcid.org/0000-0002-6490-4821
Pamela Mendoza Montes pamelamendozam@hotmail.com
Universidad Anáhuac, México
(D) https://orcid.org/0000-0002-3295-1295

Resumen: Dos estudios evaluaron la capacidad de cuestionarios basados en la Teoría de la Sensibilidad al Reforzador (TSR) para predecir psicopatía primaria y secundaria. Los instrumentos se aplicaron a muestras no probabilísticas de estudiantes universitarios (primer estudio $\mathrm{n}=769$; segundo $\mathrm{n}=528$ ). Las muestras se reunieron en universidades de la Ciudad de México. Con la finalidad de identificar predictores de las variables dependientes se usaron modelos de regresión lineal, inferencia no paramétrica y correlacionales. El primer estudio mostró que las escalas BIS/BAS (Carver \& White, 1994) predicen ambas psicopatías. La psicopatía primaria es predicha por una combinación de razgos BIS y BAS; por su parte, solamente la escala BAS predice psicopatía secundaria. El segundo estudio mostró que el cuestionario RST-PQ (Corr \& Cooper, 2016) predice ambas psicopatías. Tanto psicopatía primaria como secundaria, son predichas por una combinación de escalas pertenecientes a los sistemas de inhibición, activación conductual y FFFS. Los hallazgos no apoyan la idea de que cada una de las psicopatías se encuentre asociada, de manera independiente, a alguno de los sistemas de la TSR.

Palabras clave: Teoría de la sensibilidad al reforzador, psicopatía, conducta delincuencial, cuestionarios, estudiantes universitarios.

Abstract: Two studies assessed the capacity of self-report questionnaires, based on Reinforcement Sensitivity Theory (RST) to predict primary and secondary psychopathy. The scales were answered by a non-probabilistic sample of college students (first study, $\mathrm{n}=769$; second study, $\mathrm{n}=528$ ). Both samples were obtained from universities in Mexico City. To identify predictors of the dependent variables, linear regression, nonparametric inference tests and correlational models were used. The first study showed that the BIS/BAS scales (Carver \& White, 1994) might possess subscales that predict both psychopathy categories. Primary psychopathy is predicted by a combination of BIS and BAS subscales and only some BAS subscales predict secondary psychopathy. The second study showed that the RST-PQ questionnaire (Corr \& Cooper, 2016) might also 
possess subscales that predict both psychopathy categories. Both primary and secondary psychopathy are predicted by a combination of scales from the behavior activation, FFFS and inhibition domains. Results suggest that the idea that each psychopathy belongs to independent TSR systems is not supported by data.

Keywords: Reinforcement sensitivity theory, psychopathy, delinquent behavior, questionnaires, college students.

\section{Introducción}

Durante las décadas de los setentas y ochentas del siglo pasado, empezaron a emerger teorías de la personalidad basadas en diseños experimentales y usando sujetos no humanos como sujetos. Por ejemplo, Gray $(1970,1982)$ llevó a cabo diferentes experimentos usando para ello el modelo de supresión instrumental condicionada en ratas (Estes \& Skinner, 1941). Sus resultados sugirieron que el procedimiento se aprendía de forma más rápida por algunos individuos (y más lenta con otros). Complementariamente, fármacos que inhibían selectivamente la actividad septo hipocampal del cerebro, modulaban el desempeño en el procedimiento experimental. Además, la zona del cerebro en cuestión parecía más desarrollada en algunos individuos (específicamente en aquellos que aprendían más rápido el procedimiento). A partir de sus resultados, Gray (1987) concluyó que los roedores podrían diferir en su capacidad para condicionarse a estímulos aversivos, y que dicha capacidad estaba determinada por su química y arquitectura cerebral; concluyó igualmente que la característica era relativamente estable, a lo largo de la vida del animal. A partir de sus estudios, planteó la Teoría de la Sensibilidad al Reforzamiento (TSR). En sus orígenes, la TSR planteaba que los organismos poseen dos sistemas motivacionales, mismos que son activados por estímulos diferentes. Aquellos estímulos asociados con castigo y/o ausencia de reforzamiento, activan el sistema de inhibición conductual (BIS por sus siglas en inglés). Complementariamente, estímulos asociados con reforzamiento "ponen en movimiento" el sistema de activación conductual (BAS por sus siglas en inglés). Al continuar sus experimentos con seres humanos (Gray, 1981; Gray \& McNaughton, 1996, 2000) los datos sugirieron la generalidad entre especies de la teoría. Iniciaron igualmente un interés por desarrollar instrumentos de auto reporte que permitieran clasificar a los individuos en base a la dicotomía BIS/BAS. De dicho esfuerzo dos instrumentos han destacado por sus propiedades psicométricas (véase Corr, 2016 para una revisión). Por un lado, las escalas BIS/BAS de Carver y White (1994), por otro el SPSRQ, elaborado por Torrubia et al. (2001). A partir de esas escalas ha sido posible obtener puntajes cuantitativos de BIS y de BAS; las escalas también han permitido evaluar supuestos teóricos de la TSR. Por ejemplo, puntajes altos de BAS implican que el sistema de activación conductual del individuo se "enciende" fácilmente, (y de manera intensa), en presencia de estímulos reforzantes; dicha activación "vuelve al sujeto impulsivo" y puede llevarlo a tomar malas decisiones (Franken \& Muris, 2005). En base a la lógica anterior, diversos estudios han demostrado que puntajes altos de BAS correlacionan de manera 
directa y significativa con impulsividad (Franken et al., 2005; Leone \& Russo, 2009; Russo et al., 2008). Complementariamente, puntajes altos de BAS están asociados al abuso de alcohol (Studer et al., 2016; Wardell et al., 2011) la ludopatía (Farrell \& Walker, 2019; Gaher et al., 2015) y el comer de manera compulsiva (Loxton \& Tipman, 2017; Schienle et al., 2009). Por otro lado, puntajes altos de BIS implican que el sistema de inhibición conductual se activa, rápida e intensamente, ante estímulos asociados con castigo. Dicha activación podría desencadenar una reacción de ansiedad (Segarra et al., 2007) o depresión (Pinto-Meza et al., 2007), mismas que orientarían al sujeto a la manifestación de conductas evitativas. En base a esta lógica, diversos estudios han mostrado correlaciones directas y significativas entre puntajes altos de BIS y la conducta fóbica (Coplan et al., 2006; Kimbrel, 2008; Kramer et al., 2015).

Como puede apreciarse en los párrafos anteriores, la TSR y sus escalas asociadas han sido empleadas con frecuencia para predecir diversos tipos de problemas y trastornos conductuales (véase Bijttebier et al., 2009 para una revisión). La psicopatía no ha sido la excepción, ya que se encuentra estrechamente vinculada con la actividad criminal (Hare \& Hart, 1993). Estudiar ambos fenómenos es relevante, debido al aumento de los índices de criminalidad en México. Gray (1970) sugirió que la psicopatía es básicamente el resultado de una falta de sensibilidad al castigo y una sensibilidad "normal" al reforzamiento. Sin embargo, a lo largo de los años se ha acumulado evidencia científica que sugiere que el concepto de psicopatía puede subdividirse en diferentes tipos; y que cada uno de ellos podría corresponder a una sensibilidad BIS/BAS diferente (Skeem et al., 2003). Por ejemplo, el científico norteamericano Lykken (1995), propuso que los individuos pueden presentar dos tipos diferentes de psicopatía. Por un lado, se encuentra la psicopatía primaria. En este diagnóstico, el sujeto se caracteriza por presentar poco miedo a las consecuencias de su conducta, y las amenazas y castigos sociales tienen poco efecto sobre su comportamiento (BIS inhibido). En oposición a la psicopatía primaria, la secundaria se caracteriza por una activación intensa del BAS en presencia de estímulos reforzantes (o de aquellos que han sido asociados con reforzamiento). Newman et al. (2005) pusieron a prueba esta hipótesis en una muestra de individuos en situación de cárcel de Estados Unidos, sus resultados mostraron que los sujetos se dividían en la forma predicha por Likken. A pesar de lo anterior, otros estudios conducidos en muestras similares han fracasado en replicar el hallazgo en EU y en Bélgica (Ross et al., 2009; Uzieblo et al., 2007). Básicamente, los autores previamente citados encontraron que ambos diagnósticos de psicopatía dependen de puntajes altos de BAS (aunque en el caso de la psicopatía primaria, el diagnóstico también está asociado a puntajes BIS bajos). Dada la dificultad para obtener acceso a muestras en situación de cárcel, el modelo RST aplicado a la psicopatía también ha sido evaluado en muestras de estudiantes universitarios de EU (Hundt et al., 2008; Kimbrel et al., 2007; Ross et al., 2007). En general los resultados encontrados con este tipo de muestras también han sido mixtos. Es decir, algunos de estos estudios apoyan la hipótesis de Likken, otros sugieren 
que puntajes BAS elevados son comunes a ambas psicopatías (aunque en el caso de la psicopatía primaria también se encuentran puntajes bajos de BIS). En general el consenso en esta literatura científica, es el de que aún es necesaria más investigación para evaluar la hipótesis de Lykken. Además de aportar datos que permitan aceptar o rechazar la hipótesis en cuestión, es necesario reconocer que existe poca información acerca de las propiedades psicométricas de los cuestionarios diseñados para medir la TSR en México (como se puede ver en la revisión, casi toda la investigación viene de Estados Unidos o Europa). Así pues, el presente estudio también tiene por objetivo evaluar la validez empírica de los cuestionarios.

\section{Primer Estudio}

Las escalas BIS/BAS elaboradas por Carver y White (1994) para población de EU, fueron adaptadas recientemente a población universitaria mexicana. Pulido et al. (2016a) mostraron que dichas escalas poseen validez concurrente y consistencia interna aceptables. Por su parte, Pulido et al. (2016b) confirmaron los datos anteriores, y además demostraron que las escalas permiten predecir consumo problemático de alcohol y tabaco. Dado que el comportamiento de las escalas ha sido aceptable en muestras universitarias mexicanas, el objetivo del presente estudio es evaluar el modelo TSR de Lykken (1995), usando las escalas en cuestión. Si se encuentra que el único predictor significativo de psicopatía primaria es BIS, y complementariamente que dicho predictor mantiene una relación inversa con la variable dependiente, habrá razones para aceptar las ideas de Lykken sobre psicopatía primaria. Complementariamente si el único predictor de psicopatía secundaria es BAS, y dicho predictor mantiene una relación directa con la variable de interés, el modelo de Lykken podrá ser aceptado en su totalidad (es decir tanto en lo concerniente a psicopatía primaria como secundaria).

\section{Método}

\section{Participantes}

Un total de 769 estudiantes de licenciatura de tres diferentes campus de una universidad de la Ciudad de México, participaron en el estudio. Predominaron las estudiantes de sexo femenino (61.7\%) y el promedio de edad fue de 20.7 años, con una desviación estándar de .64 años. Predominaron los estudiantes de primer, tercer y quinto semestre (respectivamente $19.6 \%, 18 \%$ y $16.5 \%$ ). La mayor parte de los estudiantes vivía con sus padres $(86.2 \%)$. 


\section{Instrumentos}

Se utilizaron las escalas BIS/BAS desarrolladas por Carver y White (1994) para población de EU y adaptadas para estudiantes universitarios mexicanos por Pulido et al. (2016a). La escala BIS/BAS consta de 20 afirmaciones que se contestan mediante una escala tipo Likert de cuatro opciones que van desde "Totalmente de acuerdo" hasta "Totalmente en desacuerdo". Los reactivos se agrupan en dos grandes sub-escalas: (a) Sistema de Inhibición Conductual (BIS) con 7 reactivos y (b) Sistema de Activación Conductual (BAS) con 13 reactivos. La escala BAS se divide a su vez en: (a) Búsqueda de emociones (4 reactivos), (b) Responsividad (5 reactivos) y (c) Motivación (4 reactivos). "Búsqueda de emociones" es una sub escala que contiene reactivos que pretenden evaluar el grado en que el individuo reporta que disfruta de nuevas actividades y situaciones. Por su parte, "responsividad" contiene reactivos que evalúan el grado en que el individuo disfruta de actividades o situaciones "reforzantes". Finalmente, "motivación" es una sub escala que contiene reactivos que evalúan la persistencia con la que el individuo persigue actividades o situaciones reforzantes. En lo concerniente a BIS, los reactivos pretenden evaluar el grado en que el individuo acepta que el castigo tiene un efecto inhibitorio sobre su comportamiento. En el presente estudio, el instrumento mostró una consistencia interna, medida mediante alfa de Cronbach, de .764.

Por ser la escala más empleada en la literatura de interés para este estudio (véase Hughes et al., 2012), se empleó para medir psicopatía, la "Escala de Auto Reporte de Levenson". La escala cuenta con veintiséis reactivos que permiten medir psicopatía primaria y secundaria. Dieciséis de estos miden psicopatía primaria y los diez restantes psicopatía secundaria. En el presente estudio, la escala tuvo una consistencia interna global de .827. La escala se contesta mediante una escala tipo Likert de cinco opciones, mismas que oscilan en un rango entre "completamente de acuerdo" y hasta "completamente en desacuerdo". La escala fue traducida del inglés al español mediante tres jueces completamente bilingües, usando la técnica de traducción-retraducción.

\section{Procedimiento}

Antes de iniciar el semestre, se sometió a consideración de la Dirección de investigación de la facultad de psicología de la Universidad Anáhuac, el presente proyecto. La Dirección aprobó el proyecto y la carta de consentimiento informado. La carta establecía que la participación sería voluntaria, no existiría pago alguno a los participantes (y que la información personal de los participantes sería confidencial). Los instrumentos se aplicaron en grupos naturales de los campus muestreados, en aquellos salones donde tanto los alumnos como el docente aceptaron participar. Después de presentarse, los aplicadores explicaron que la participación en la investigación era totalmente voluntaria; que la decisión de no participar no tendría consecuencias para los estudiantes, (y que la identidad de los participantes sería guardada en anonimato). 
Esta información también fue presentada por escrito, a través de una carta de consentimiento informado. A los estudiantes que aceptaron participar y que firmaron la carta, se les repartió una batería impresa que tenía los instrumentos descritos previamente; se les solicitó que, al terminar de contestar, doblaran el cuadernillo y lo colocaran en una urna de cartón ubicada al frente del salón. Una vez aplicados los cuestionarios, los datos fueron capturados y analizados en utilizando el programa SPSS versión 20. Solamente fueron considerados para el análisis cuestionarios completos; complementariamente, aquellos cuestionarios carentes de datos demográficos fueron descartados.

\section{Resultados}

El lector podrá ver que en los presentes estudios se combina la información proporcionada por modelos paramétricos y no paramétricos. Hacerlo permite ver los datos desde diferentes puntos de vista. Identificar y evaluar predictores estadísticos en base a las congruencias entre los modelos. La estrategia ha sido debatida en profundidad; se ha encontrado que tiene ventajas y desventajas (pero difícilmente es una práctica nueva, véase por ejemplo Wey et al., 2015). La primera tabla muestra la correlación de Pearson entre las escalas BIS/BAS, y los puntajes obtenidos en psicopatía primaria y secundaria a través de la escala de Levenson.

Tabla 1

Tabla 1

Correlaciones de Pearson. Escalas BIS/BAS vs Psicopatía.

\begin{tabular}{lll}
\hline Escalas BIS/BAS & Psicopatía & Psicopatía \\
& Primaria & Secundaria \\
\hline BIS & $\mathrm{r}=-.223^{* *}$ & $\mathrm{r}=.050$ \\
BAS-R & $\mathrm{r}=-.134^{* *}$ & $\mathrm{r}=-.053$ \\
BAS-B & $\mathrm{r}=.137^{* *}$ & $\mathrm{r}=.150^{* *}$ \\
BAS-M & $\mathrm{r}=.224^{* *}$ & $\mathrm{r}=.118^{* *}$
\end{tabular}

${ }^{*} p \leq .05 ;{ }^{* *} p \leq .01$

$$
{ }^{*} \mathrm{p}<.05
$$

Como se puede observar en la tabla, todas las correlaciones entre las escalas BIS/BAS y psicopatía primaria son estadísticamente significativas. Dos de ellas son correlaciones directas (Bas-Búsqueda y Bas-Motivación); las restantes son correlaciones inversas. En lo correspondiente a psicopatía secundaria, solamente Bas-Búsqueda y Bas-Motivación correlacionan de 
manera estadísticamente significativa con la variable en cuestión. Ambas correlaciones son directas.

La Tabla 2 muestra regresiones lineales múltiples entre las escalas BIS/BAS (como variables independientes) y los puntajes obtenidos de psicopatía primaria y secundaria (como variables dependientes).

Tabla 2

Tabla 2

Regresión Lineal Múltiple entre las Escalas BIS/BAS y Psicopatía

\begin{tabular}{|c|c|c|c|c|c|c|c|}
\hline Escalas & $\beta$ & $t$ & $p$ & Escalas & $\beta$ & $t$ & $p$ \\
\hline Psicopatía Primaria & & & & Psicopatía Secundaria & & & \\
\hline BAS-R & -.24 & -5.95 & $.00 * *$ & BAS-R & -.20 & 4.81 & $.00 * *$ \\
\hline BAS-B & .09 & 2.27 & $.02 *$ & BAS-B & .20 & 4.75 & $.00 * *$ \\
\hline BAS-M & .26 & 6.8 & $.00 * *$ & BAS-M & .11 & 2.78 & $.00 * *$ \\
\hline BIS & -.16 & -4.52 & $.00 * *$ & BIS & .12 & 3.22 & $.00 * *$ \\
\hline Constante & & 10.28 & $.00 * *$ & Constante & & 7.06 & $.00 * *$ \\
\hline
\end{tabular}

Como puede verse en la Tabla 2, todas las escalas BIS/BAS son predictores estadísticamente significativos de psicopatía. En el caso de la psicopatía primaria, los mejores predictores (basándose en los valores del coeficiente de regresión estandarizado) son BAS-M y BAS-R. Dos de los predictores mantienen relaciones negativas con psicopatía primaria (BAS-R y BIS). En lo concerniente a psicopatía secundaria, los mejores predictores son BAS-R y BAS-B. Solamente BAS-R mantiene relación negativa con psicopatía secundaria.

La Tabla 3 muestra, como "variable de agrupación", los cuartiles obtenidos de los puntajes brutos de las escalas BIS/BAS; como variable dependiente se muestra el rango promedio de psicopatía primaria obtenido para cada cuartil. Las frecuencias en las categorías se compararon usando una prueba no paramétrica de Kruskal-Wallis. 
Tabla 3

\section{Tabla 3}

Escalas BIS/BAS vs Psicopatía Primaria

\begin{tabular}{lllll}
\hline Cuartil & BIS & BAS-R & BAS-B & BAS-M \\
\hline 1 & 422.1 & 192.8 & 162.3 & 228.0 \\
2 & 400.9 & 217.7 & 246.4 & 260.8 \\
3 & 342.7 & 261.3 & 254.0 & 267.4 \\
4 & 315.2 & 290.6 & 291.7 & 317.6 \\
\hline$K-W$ & $32.6^{* *}$ & $33.16^{* *}$ & $48.63^{* *}$ & $21.97^{* *}$ \\
\hline$n$ & 730 & 727 & 729 & 730 \\
\hline$* \leq .05 ; * * p \leq .01$ & & &
\end{tabular}

Como puede verse en la Tabla, las frecuencias de psicopatía primaria en los cuartiles de las escalas BIS/BAS, difieren estadísticamente. Con la excepción de la escala BIS, en las restantes el rango promedio aumenta con cada cuartil.

La Tabla 4 muestra una agrupación idéntica a la 3, sin embargo, ahora la variable dependiente lo constituyen los rangos promedio de psicopatía secundaria obtenidos para cada cuartil. Al igual que en la tabla anterior, las frecuencias en las categorías se compararon usando una prueba no paramétrica de Kruskal-Wallis. 
Tabla 4

Tabla 4

Escalas BIS/BAS vs Psicopatía Secundaria

\begin{tabular}{lllll}
\hline Cuartil & BIS & BAS-R & BAS-B & BAS-M \\
\hline 1 & 366.5 & 406.7 & 346.9 & 354.8 \\
2 & 365.2 & 371.1 & 330.4 & 364.8 \\
3 & 352.3 & 343.4 & 407.5 & 353.7 \\
4 & 397.2 & 378.4 & 402.6 & 408.5 \\
\hline$K-W$ & 5.22 & $8.24 *$ & $21.24^{* *}$ & $9.54^{*}$ \\
\hline$n$ & 745 & 743 & 744 & 744 \\
${ }^{*} \leq \leq .05 ;{ }^{* *} p \leq .01$ & & &
\end{tabular}

Como puede verse en la Tabla, las frecuencias de psicopatía secundaria en los cuartiles difieren de forma estadísticamente significativa para todas las escalas BAS. Con la excepción de la escala BAS-R, en las restantes el rango promedio tiende a aumentar con cada cuartil.

\section{Discusión}

Los datos obtenidos en el presente estudio fueron analizados con tres modelos estadísticos diferentes. Los tres modelos arrojan resultados congruentes en lo concerniente a psicopatía primaria. En síntesis, todas las escalas BIS/BAS son predictores de psicopatía primaria. Al parecer la psicopatía primaria se puede predecir a través de puntajes bajos de BIS y BAS-R; complementariamente la psicopatía primaria se predice a través de puntajes altos de BAS-B y BAS-M. En lo concerniente a la psicopatía secundaria, los tres modelos estadísticos empleados sugieren una relación directa entre BAS-B, BAS-M y la variable en cuestión. Los modelos empleados son menos consistentes en lo concerniente al papel que pueden jugar BAS-R y BIS en la predicción de psicopatía secundaria. En síntesis, los resultados obtenidos no son congruentes con la hipótesis de Lykken (1995) en el sentido de que cada una de las dos psicopatías es predicha, exclusivamente, por una de las dimensiones de la TSR. Los resultados del presente estudio tampoco coinciden con los obtenidos por Newman et al. (2005) en una muestra de 517 individuos en situación de cárcel de EU. Dichos autores reportan que los sujetos con diagnóstico de psicopatía primaria, se identifican exclusivamente en base a puntajes muy bajos de BIS; complementariamente, el único predictor consistente de psicopatía secundaria son puntajes altos de 
BAS. Este último resultado de Newman y colegas es difícil de evaluar, debido a que no analizan por separado las diferentes sub escalas de BAS. Los resultados obtenidos en el presente estudio tampoco coinciden con los reportados por Hundt et al. (2008) en una muestra de 273 estudiantes de licenciatura de EU. Hundt y colegas encontraron que las únicas correlaciones estadísticamente significativas de su estudio, fueron aquellas entre BAS y psicopatía primaria y secundaria. En ambos casos, las correlaciones fueron directas. De nueva cuenta, los resultados de los investigadores son difíciles de evaluar, debido a que usaron una medición agrupada de BAS (así pues, no es posible identificar si las tres sub escalas de BAS mantienen correlaciones con las psicopatías).

Los datos obtenidos en el presente estudio coinciden con los obtenidos por Kimbrel et al. (2007). Empleando una muestra de 181 estudiantes universitarios de EU, los autores encontraron correlaciones directas entre psicopatía primaria y BAS; inversas y significativas entre esta misma variable y BIS. Complementariamente encontraron correlaciones directas y significativas entre BAS y psicopatía secundaria. Sin embargo, la coincidencia entre los resultados de Kimbrel y colegas y los datos aquí reportados es difícil de establecer, pues también emplearon una medición agregada de BAS. Ross et al. (2007), usando una muestra de 326 estudiantes de licenciatura de EU, llegaron a las mismas conclusiones que Kimbrel y colaboradores (y a la misma conclusión arrojada por el presente estudio). Ross y colegas también usaron una medición agregada de BAS.

En síntesis, los resultados del presente estudio, apoyan la idea de puntajes bajos de BIS y altos de BAS, podrían ser los mejores predictores de psicopatía primaria. Complementariamente, puntajes altos de BAS podrían ser predictores de psicopatía secundaria. Los resultados obtenidos, así como los reportados por Kimbrel et al. (2007) y Ross et al. (2007) no apoyan la hipótesis de Lykken planteada en 1995. Tampoco ofrecen una razón contundente que permita explicar las discrepancias). Además de aportar evidencia sobre la hipótesis de Lykken, el presente estudio también permite un análisis de cada una de las correlaciones entre las diferentes sub escalas que constituyen al BAS, y las psicopatías. Al parecer, BAS-R tiene un comportamiento sustancialmente diferente a las otras escalas que constituyen al BAS. Se comporta de forma similar al BIS, al predecir psicopatía primaria y secundaria. El hallazgo sugiere pues que usar las tres escalas del BAS como una medida agregada, podría carecer de sustento empírico (y podría llevar a realizar predicciones equivocada de las psicopatías). La falta de correlación entre BAS-R y las restantes escalas del BAS, no debe ser ignorado, ya que ha sido reportado por otros investigadores (véase, por ejemplo, Corr, 2008; Voigt et al., 2009). De hecho, los problemas aquí reportados por el constructo BAS, así como otros reportados por el constructo BIS (véase por ejemplo; Corr \& McNaughton, 2012; Gray \& McNaughton, 2000; McNaughton \& Corr, 2004, 2008) han llevado a revisar la TSR. El segundo estudio que aquí se presenta aborda la Teoría de la Sensibilidad Revisada (r-TSR) y la emplea para hacer predicciones de las psicopatías. La $\mathrm{r}$-TSR ha desarrollado sus propios (y nuevos) instrumentos de medición; 
la capacidad de estas herramientas para predecir psicopatía primaria y secundaria se evalúa en el segundo estudio. Además de evaluar la capacidad predictiva de la r-TSR, el segundo estudio también evalúa la validez empírica de un cuestionario diseñado para medirla.

\section{Segundo Estudio}

Gray y McNaughton (2000) publicaron una revisión de estudios en la cual se evaluaba el comportamiento de ratas en preparaciones en las cuales un espacio accesible al roedor contenía alimento y también un depredador (Blanchard et al., 1990). En general, los resultados de los estudios mostraron que aquellas ratas que mostraban "comportamiento de miedo" (pelear, huir, congelamiento) en la preparación no siempre presentaban conductas relacionadas con ansiedad (por ejemplo, vocalizaciones de advertencia). Los resultados llevaron a estos autores a revisar la TSR (r-TSR). En dicha revisión se plantea que al menos dos sistemas ayudan a los organismos a enfrentar estímulos aversivos. El primero de ellos es el sistema FFFS (sistema de pelea, fuga congelamiento). El segundo es el sistema BIS; dicho sistema se activa en presencia de estímulos conflictivos (es decir ante estímulos que incluyen tanto estímulos reforzantes como aversivos). En congruencia con los datos obtenidos en roedores, Cooper et al. (2007) llevaron a cabo análisis factoriales confirmatorios (AFC) en instrumentos diseñados para medir BIS de acuerdo con el modelo TSR original. Sus resultados mostraron que los modelos consistentemente dividían los reactivos de dicha escala en dos, categorías (reactivos relacionados con ansiedad y aquellos relacionados con miedo). Resultados similares se han obtenido dentro de la literatura científica relacionada con ansiedad en población belga (Bijttebier et al., 2009). Es decir, con frecuencia los AFC dividen los reactivos en dos categorías, ansiedad y miedo. Las razones mencionadas, y otras (Perkins et al., 2012) han llevado a algunos científicos a aceptar la r-TSR (véase McNaughton \& Corr, 2008 para una revisión). Los resultados también sugieren que escalas que no dividen el factor BIS en ansiedad y miedo fracasan en evaluar apropiadamente los constructos que subyacen a la r-TSR (véase Corr [2016] para una revisión). En los últimos años han surgido escalas que pretenden medir r-TSR (véase Jackson, 2009; Reuter et al., 2015; Smederevac et al., 2014). Desafortunadamente ninguna de ellas segmenta las escalas BAS, y los datos obtenidos en el primer estudio (y otros, véase, por ejemplo, Voigt et al., 2009) sugieren que no todas las sub escalas del BAS se comportan del mismo modo. Corr y Cooper (2016) desarrollaron el RST-PQ. El instrumento subdivide el BIS es la forma sugerida por r-TSR; complementariamente el BAS se subdivide de una forma similar a la sugerida por Carver y White en 1994. Por estos motivos, los presentes autores consideran que el RST-PQ puede ser una mejor herramienta que las escalas BIS/BAS para medir la personalidad de los individuos. En sintonía con el primer estudio, el objetivo del segundo fue usar dicho instrumento para predecir psicopatía primaria y secundaria. Complementariamente, la psicopatía medida en estudiantes 
universitarios, no siempre se traduce en conducta delincuencial. Así pues, con la finalidad de evaluar la capacidad del RST-PQ para medir esta variable, se incluyó en la batería aplicada, una lista de cotejo para evaluar diversas conductas delincuenciales. Esta fue tomada del Cuestionario de Conducta Antisocial elaborado por Luengo et al. (1999).

\section{Método}

\section{Participantes}

Un total de 528 estudiantes universitarios de la Ciudad de México participaron en el estudio. La mayor parte de los participantes fueron de sexo masculino (60.4\%) y su edad promedio fue de 20.8 años con una desviación estándar de 2.1 años. Participaron estudiantes de diferentes licenciaturas y semestres. La mayor parte de los estudiantes vivía con su familia original. La participación fue voluntaria, previa firma de la carta de consentimiento informado.

\section{Instrumentos}

Los participantes recibieron una batería que contenía los siguientes elementos: a) Una carta de consentimiento informado, (idéntica a la empleada en primer estudio), b) El RST-PQ elaborado por Corr y Cooper (2016) traducido al español, c) La Escala de Autoreporte de Levenson descrita en el primer estudio y d) Una lista de cotejo sobre "conductas delincuenciales" que son frecuentes en estudiantes universitarios. El RST-PQ fue construido por Corr y Cooper (2016) como un instrumento de auto reporte que permitiera evaluar la r-TSR. Consta de 79 afirmaciones, y se pide al participante que evalúe, en una escala tipo Likert, "qué tan bien lo describen las afirmaciones en cuestión". Las opciones de respuesta son: "nada en lo absoluto", "un poco", "moderamente" y "mucho". La escala fue construida en cuatro etapas. En la primera, un grupo de individuos contestaron un cuestionario de "situaciones de vida", mismo que dio lugar a un grupo focal dirigido. A partir del análisis cualitativo de las respuestas de los participantes, se procedió a construir reactivos. Dichos reactivos fueron analizados, primero, mediante análisis de extracción de factores exploratorio (AFE). Los mejores reactivos obtenidos del AFE fueron sometidos a un análisis de extracción de factores confirmatorio (AFC), mismo que sirvió como un último filtro de reactivos. Ya con la versión definitiva del instrumento se procedió a realizar un análisis de validez concurrente con diferentes instrumentos diseñados para medir RST y r-RST. Entre los principales hallazgos de Corr y Cooper (2016) están los siguientes. Los reactivos relacionados con comportamiento ante estímulos aversivos se dividen en dos factores, el primero de ellos tiene que ver con ansiedad (BIS); el segundo con miedo (sistema de pelea, huida o "congelamiento, FFFS por sus siglas en inglés). En cuanto al BIS, se hipotetiza como un sistema 
que opera cuando simultáneamente se presentan estímulos aversivos y reforzantes. Ante esta disyuntiva el organismo tiene que realizar una valoración extraordinaria de los aspectos peligrosos (y deseables) de la situación. Se asume que la principal emoción asociada a la activación del BIS es la ansiedad. Por su parte el FFFS se conceptualiza como un sistema de evitación del castigo. Cuando puede evitarse fácilmente, se activa "huida" (flight); conforme se vuelve más difícil evitar el estímulo punitivo, se activan "pelea" (fight) y "congelamiento" (freeze), en ese orden. El RST-PQ también contiene dos escalas derivadas de FFFS. Se trata de las escalas PA (Panic Attack) y DF (defensive fighting). Las escalas fueron desarrolladas debido a que, de acuerdo con Corr y Cooper (2016), el sistema FFFS no alcanza a explicar, las reacciones de miedo extremo (PA) o la pelea defensiva en seres humanos (DF). Finalmente, los reactivos relacionados con el BAS se dividieron en cuatro categorías: a) Interés en la recompensa (Reward Interest, o RI por sus siglas en inglés), b) Persistencia en la búsqueda de la recompensa (Goal Drive Persistence, o GDP), c) Efectos de la recompensa (Reward Responsivity, o RR) e d) Impulsividad (Impulsivenes, o IMP). En cuanto a RI, está conformado por reactivos que tienen que ver con un interés por vivir nuevas experiencias (oportunidades de reforzamiento). Se hipotetiza que es un sistema que opera en ausencia del estímulo reforzante (expectativa anticipatoria). Por su parte GDP contiene reactivos que evalúan la capacidad del sujeto de planear y demorar la recompensa. De "persistir en la búsqueda de la meta a través de los estímulos condicionados" (Corr \& Cooper, 2016). En lo concerniente a RR, se refiere reactivos que miden el "goce o disfrute" que produce el estímulo incondicionado, "el placer provocado por alcanzar la recompensa”. Finalmente, IMP contiene reactivos que miden el comportamiento del individuo al aproximarse al estímulo consumatorio. Es decir, una vez que la recompensa está "a la mano", el individuo rápidamente se apropia de él (o no). En el presente estudio el RST-PQ mostró una consistencia interna, medida mediante alfa de Cronbach de .891 . La traducción del instrumento se realizó mediante la técnica de traducción retraducción, por tres jueces completamente bilingües. En total el RST-PQ consta de 79 reactivos (considerando también las escalas añadidas PA y DF).

En lo concerniente a la lista de cotejo de conductas delincuenciales. Esta fue tomada del Cuestionario de Conducta Antisocial elaborado por Luengo et al. (1999). El instrumento está diseñado para medir conducta antisocial en adolescentes y adultos jóvenes. Ha mostrado ser válida y confiable en población española y brasileña. En la presente investigación mostró una consistencia interna, medida mediante alfa de Cronbach de .787. La escala original consta de 60 ítemes; para el presente estudio se tomaron solamente aquellos quince que miden las conductas que con mayor frecuencia se presentan en la Universidad muestreada. En la escala se pide al participante que conteste si ha llevado a cabo (o no) la conducta, (por ejemplo, "pelearse con una persona a golpes"). 


\section{Procedimiento}

El procedimiento empleado en el segundo estudio fue idéntico al descrito para el primero. Antes de iniciar el semestre, se sometió a consideración de la Dirección de investigación de la facultad de psicología de la Universidad Anáhuac, el presente proyecto. La Dirección aprobó el proyecto y la carta de consentimiento informado. La carta establecía que la participación sería voluntaria, no existiría pago alguno a los participantes (y que la información personal de los participantes sería confidencial). Solamente aquellos alumnos que aceptaron contestar el cuestionario, y firmaron la carta de consentimiento informado participaron en el estudio.

\section{Resultados}

El lector podrá ver que en los presentes estudios se combina la información proporcionada por modelos paramétricos y no paramétricos. Hacerlo permite ver los datos desde diferentes puntos de vista. Identificar y evaluar predictores estadísticos en base a las congruencias entre los modelos. La estrategia ha sido debatida en profundidad; se ha encontrado que tiene ventajas y desventajas (pero difícilmente es una práctica nueva, véase por ejemplo Wey et al., 2015). La Tabla 5 muestra las correlaciones de Pearson entre las sub escalas del RST-PQ con psicopatía primaria, secundaria y conducta delincuencial.

\section{Tabla 5}

\section{Tabla 5}

Correlaciones de Pearson. Escalas de RST-PQ vs Psicopatia y C. Delincuencial

\begin{tabular}{llll}
\hline Escalas & Psicopatía & Psicopatía & C. Delincuencial \\
BIS/BAS & Primaria & Secundaria & \\
\hline BIS & $\mathrm{r}=.062$ & $\mathrm{r}=.289^{* *}$ & $\mathrm{r}=-.006$ \\
RR & $\mathrm{r}=.071$ & $\mathrm{r}=.007$ & $\mathrm{r}=-.037$ \\
GDP & $\mathrm{r}=.030$ & $\mathrm{r}=-.104$ & $\mathrm{r}=-.075$ \\
DF & $\mathrm{r}=.307^{* *}$ & $\mathrm{r}=.179 * *$ & $\mathrm{r}=.201 * *$ \\
FFFS & $\mathrm{r}=.037$ & $\mathrm{r}=.187^{* *}$ & $\mathrm{r}=.042$ \\
RI & $\mathrm{r}=.041$ & $\mathrm{r}=-.037$ & $\mathrm{r}=-.053$ \\
PA & $\mathrm{r}=.109 *$ & $\mathrm{r}=.269 * *$ & $\mathrm{r}=.111^{*}$ \\
IMP & $\mathrm{r}=.234^{* *}$ & $\mathrm{r}=.305^{* *}$ & $\mathrm{r}=.070$
\end{tabular}

${ }^{*} p \leq .05 ; * * p \leq .01$

Como puede verse en la Tabla 5, hay dos correlaciones directas y estadísticamente significativas que comparten las psicopatías (primaria y 
secundaria) y la conducta delincuencial, se trata de DF y PA. Por su parte, las psicopatías comparten tres correlaciones directas y estadísticamente significativas, se trata de DF, PA e IMP. Finalmente hay correlaciones directas y estadísticamente significativas que únicamente aparecen en psicopatía secundaria, son BIS y FFFS.

La Tabla 6 muestra los resultados de un análisis de regresión lineal múltiple entre las diferentes escalas del RST-PQ, como variables independientes, y la psicopatía (primaria columna izquierda y secundaria columna derecha) como variables dependientes.

Tabla 6

\section{Tabla 6}

Regresión Lineal Múltiple entre las Escalas del RST-PQ y Psicopatia

\begin{tabular}{|c|c|c|c|c|c|c|c|}
\hline Escalas & $\beta$ & $t$ & $p$ & Escalas & $\beta$ & $t$ & $p$ \\
\hline Psicopatía Primaria & & & & Psicopatía Secundaria & & & \\
\hline BIS & -.06 & -1.05 & .29 & BIS & .12 & 2.21 & $.03 *$ \\
\hline $\mathrm{RR}$ & -.05 & -1.00 & .32 & $\mathrm{RR}$ & -.08 & -1.40 & .16 \\
\hline GDP & .04 & .72 & .47 & GDP & -.07 & -1.36 & .17 \\
\hline FFFS & .27 & 5.79 & $.00^{* *}$ & FFFS & .02 & .35 & .73 \\
\hline $\mathrm{DF}$ & -.01 & -.12 & .91 & $\mathrm{DF}$ & .12 & 2.57 & $.01 *$ \\
\hline RI & -.09 & -1.69 & .09 & $\mathrm{RI}$ & -.11 & -2.15 & $.03 *$ \\
\hline PA & .07 & 1.25 & .21 & PA & .11 & 2.01 & $.04 *$ \\
\hline IMP & .21 & 4.13 & $.00^{* *}$ & IMP & .27 & 5.53 & $.00 * *$ \\
\hline Constante & & 3.31 & $.00^{* *}$ & Constante & & 5.59 & $.00 * *$ \\
\hline
\end{tabular}

${ }^{*} p \leq .05 ;{ }^{* *} p \leq .01$

Como puede observarse en la Tabla 6 , hay dos predictores positivos y estadísticamente significativos de psicopatía primaria. Se trata de FFFS e IMP. En contraste, psicopatía secundaria presenta cinco predictores estadísticamente significativos, cuatro de ellos son positivos (BIS, DF, PA e IMP) y uno negativo (RI).

La Tabla 7 muestra el análisis de regresión lineal múltiple entre las diferentes escalas del RST-PQ (como variables independientes) y la conducta delictiva como variable dependiente. 
Tabla 7

Tabla 7

Regresión Lineal Múltiple entre las Escalas del RST-PQ y C. Delincuencial

\begin{tabular}{|c|c|c|c|}
\hline Escalas & $\beta$ & $t$ & $p$ \\
\hline \multicolumn{4}{|c|}{ Conducta Delincuencial } \\
\hline BIS & -.12 & -2.02 & $.04 *$ \\
\hline $\mathrm{RR}$ & -.02 & -.38 & .71 \\
\hline GDP & .03 & .56 & .58 \\
\hline FFFS & -.02 & -.31 & .76 \\
\hline DF & .19 & 3.94 & $.00 * *$ \\
\hline RI & -.11 & -2.00 & $.04 *$ \\
\hline PA & .13 & 2.13 & $.03^{*}$ \\
\hline IMP & .07 & 1.39 & .17 \\
\hline Constante & & 3.31 & $.00^{* *}$ \\
\hline
\end{tabular}

${ }^{*} p \leq .05 ;{ }^{* *} p \leq .01$

Como se puede observar en las Tabla 7 , hay cuatro predictores estadísticamente significativos de la conducta delincuencial. Dos de ellos son positivos (DF y PA) y los otros dos negativos (BIS y RI).

Las Tablas 8, 9 y 10 muestran, como variable de agrupación, los cuartiles obtenidos de los puntajes brutos de las escalas del RST-PQ, como variable dependiente se muestra el rango promedio de psicopatía primaria (Tabla 8), secundaria (Tabla 9) y conducta delincuencial (Tabla 10) obtenido para cada cuartil. Las frecuencias en las categorías se compararon usando una prueba no paramétrica de Kruskal-Wallis. 
Tabla 8

\section{Tabla 8}

Escalas del RST-PQ vs Psicopatía Primaria

\begin{tabular}{lllllllll}
\hline Cuartil & BIS & RR & GDP & DF & FFFS & RI & PA & IMP \\
\hline 1 & 241.71 & 252.73 & 257.95 & 201.25 & 262.67 & 242.16 & 243.07 & 206.21 \\
2 & 255.52 & 263.60 & 274.88 & 236.69 & 247.22 & 264.49 & 255.82 & 251.79 \\
3 & 285.10 & 259.14 & 257.22 & 280.53 & 249.20 & 266.17 & 280.96 & 254.35 \\
4 & 260.00 & 269.79 & 258.25 & 312.27 & 288.81 & 267.22 & 269.24 & 317.35 \\
\hline$K-W$ & 5.66 & .94 & 1.15 & $43.3 * *$ & 6.74 & 2.08 & 3.6 & $38.5^{* *}$ \\
\hline$n$ & 521 & 523 & 522 & 526 & 526 & 522 & 525 & 525 \\
\hline
\end{tabular}

${ }^{*} p \leq .05 ;{ }^{* *} p \leq .01$

Tabla 9

Tabla 9

Escalas del RST-PQ vs Psicopatía Secundaria

\begin{tabular}{lllllllll}
\hline Cuartil & BIS & RR & GDP & DF & FFFS & RI & PA & IMP \\
\hline 1 & 202.89 & 260.13 & 281.60 & 231.25 & 222.73 & 263.25 & 172.15 & 200.99 \\
2 & 234.78 & 280.42 & 281.32 & 243.27 & 231.08 & 249.47 & 254.96 & 246.67 \\
3 & 293.44 & 258.26 & 252.29 & 274.33 & 264.26 & 271.95 & 277.92 & 254.93 \\
4 & 309.17 & 256.73 & 239.04 & 292.14 & 319.44 & 256.83 & 308.40 & 326.38 \\
\hline$K-W$ & $42.6^{* *}$ & 1.83 & 7.64 & $14.1^{* *}$ & $34.7^{* *}$ & 1.63 & $50.1^{* *}$ & $50.2^{* *}$ \\
\hline$n$ & 522 & 524 & 523 & 527 & 527 & 522 & 526 & 526
\end{tabular}

${ }^{*} p \leq .05 ;{ }^{* *} p \leq .01$ 
Tabla 10

\section{Tabla 10}

Escalas del RST-PQ vs Conducta Delincuencial

\begin{tabular}{lllllllll}
\hline Cuartil & BIS & RR & GDP & DF & FFFS & RI & PA & IMP \\
\hline 1 & 244.06 & 251.35 & 267.00 & 220.37 & 265.19 & 261.03 & 233.96 & 232.96 \\
2 & 254.59 & 284.65 & 259.29 & 241.29 & 252.52 & 277.11 & 250.39 & 264.80 \\
3 & 299.26 & 254.11 & 262.16 & 268.10 & 267.06 & 261.05 & 284.65 & 272.56 \\
4 & 246.63 & 263.70 & 259.61 & 307.31 & 268.00 & 251.17 & 277.91 & 277.35 \\
\hline$K-W$ & $12.7 * *$ & 3.54 & .220 & $28.4 * *$ & .841 & 1.88 & $8.85^{*}$ & 7.09 \\
\hline$n$ & 522 & 524 & 523 & 527 & 527 & 522 & 526 & 526 \\
\hline
\end{tabular}

${ }^{*} p \leq .05 ;{ }^{* *} p \leq .01$

La Tabla 8 identifica dos escalas que difieren de forma estadísticamente significativa en cuanto a frecuencias observadas entre cuartiles, se trata de DF e IMP. Por su parte, la Tabla 9 muestra que hay cinco escalas en las cuales las frecuencias observadas de psicopatía secundaria difieren entre cuartiles. Se trata de BIS, DF, FFS, PA, e IMP. Finalmente la Tabla 10 muestra tres escalas en las cuales las frecuencias observadas difieren estadísticamente entre cuartiles, se trata de BIS, DF y PA.

A diferencia de las escalas BIS/BAS, sobre las que hay mucha información, el RST-PQ es relativamente nuevo. Por este motivo se presenta, en la Tabla 11, la correlación de Pearson entre las sub escalas del cuestionario, las psicopatías y la conducta delincuencial para mujeres (y Tabla 12 para hombres). 
Tabla 11

Tabla 11

Correlaciones de Pearson. RST-PQ vs Psicopatías. Mujeres

\begin{tabular}{llll}
\hline $\begin{array}{l}\text { Escalas } \\
\text { BIS/BAS }\end{array}$ & $\begin{array}{l}\text { Psicopatía } \\
\text { Primaria }\end{array}$ & $\begin{array}{l}\text { Psicopatía } \\
\text { Secundaria }\end{array}$ & C. Delincuencial \\
\hline BIS & $\mathrm{r}=.223^{* *}$ & $\mathrm{r}=.498^{* *}$ & $\mathrm{r}=.090$ \\
RR & $\mathrm{r}=.128$ & $\mathrm{r}=.080$ & $\mathrm{r}=-.105$ \\
GDP & $\mathrm{r}=-.058$ & $\mathrm{r}=-.092$ & $\mathrm{r}=-.132$ \\
DF & $\mathrm{r}=.310^{* *}$ & $\mathrm{r}=.223^{* *}$ & $\mathrm{r}=.171^{*}$ \\
FFFS & $\mathrm{r}=.262^{* *}$ & $\mathrm{r}=.424^{* *}$ & $\mathrm{r}=.154$ \\
RI & $\mathrm{r}=.047$ & $\mathrm{r}=-.026$ & $\mathrm{r}=-.135$ \\
PA & $\mathrm{r}=.244^{* *}$ & $\mathrm{r}=.442^{* *}$ & $\mathrm{r}=.223^{* *}$ \\
IMP & $\mathrm{r}=.244^{* *}$ & $\mathrm{r}=.392^{* *}$ & $\mathrm{r}=-.001$ \\
\hline
\end{tabular}

${ }^{*} p \leq .05 ;{ }^{* *} p \leq .01$

Tabla 12

Tabla 12

Correlaciones de Pearson. RST-PQ vs Psicopatías Hombres

\begin{tabular}{llll}
\hline Escalas & Psicopatía & Psicopatía & C. Delincuencial \\
BIS/BAS & Primaria & Secundaria & \\
\hline BIS & $\mathrm{r}=.010$ & $\mathrm{r}=.172^{* *}$ & $\mathrm{r}=-.056$ \\
RR & $\mathrm{r}=.053$ & $\mathrm{r}=-.039$ & $\mathrm{r}=.027$ \\
GDP & $\mathrm{r}=.042$ & $\mathrm{r}=-.132^{*}$ & $\mathrm{r}=-.049$ \\
DF & $\mathrm{r}=.263^{* *}$ & $\mathrm{r}=.129^{*}$ & $\mathrm{r}=.202^{* *}$ \\
FFFS & $\mathrm{r}=.049$ & $\mathrm{r}=.097$ & $\mathrm{r}=.011$ \\
RI & $\mathrm{r}=.032$ & $\mathrm{r}=-.048$ & $\mathrm{r}=-.011$ \\
PA & $\mathrm{r}=.089$ & $\mathrm{r}=.177^{* *}$ & $\mathrm{r}=.048$ \\
IMP & $\mathrm{r}=.226^{* *}$ & $\mathrm{r}=.244^{* *}$ & $\mathrm{r}=.120$ \\
\hline
\end{tabular}

${ }^{*} p \leq .05 ;{ }^{* *} p \leq .01$

Al comparar entre sexos, se puede ver que, para psicopatía primaria, hombres y mujeres coinciden en que ambos presentan correlaciones significativas en dos sub escalas (DF e IMP). En lo correspondiente a psicopatía secundaria, hay cuatro correlaciones significativas que coinciden para hombres y mujeres (BIS, DF, PA e IMP). Por último, 
en conducta delincuencial, hombres y mujeres presentan, ambos, correlaciones significativas para DF.

\section{Discusión}

$\mathrm{Al}$ igual que en el estudio anterior, se emplearon tres modelos estadísticos para evaluar la relación entre las escalas del RST-PQ, las “psicopatías” y la conducta delincuencial. Las conclusiones que se presentan a continuación buscan las congruencias entre los mismos. Al parecer las escalas DF e IMP (sobre todo la última) parecen predictores importantes y consistentes de psicopatía primaria. Por su parte, BIS, DF, PA, IMP y FFFS (sobretodo, las primeras cuatro) podrían ser predictores importantes y consistentes de psicopatía secundaria. Finalmente, en lo relativo a la conducta delincuencial, las escalas DF, PA (y en menor grado BIS) parecen predictores importantes de la variable en cuestión. Hay poca investigación aún sobre el RST-PQ. La mayor parte de la literatura científica sobre el instrumento, se centra en medir sus propiedades psicométricas. Los estudios existentes muestran que el instrumento posee consistencia interna aceptable (Amiri et al., 2019; Eriksson et al., 2019; Pugnaghi et al., 2018) los datos obtenidos en este estudio confirman el hallazgo. Por otro lado, un estudio realizado por Satchell et al. (2018) evaluó la capacidad del RST-PQ para evaluar toma de decisiones riesgosas. Los resultados mostraron que puntajes bajos de FFFS estaban relacionados con toma de riesgos en interacciones sociales. Complementariamente, puntajes altos de IMP estaban relacionados con toma de riesgos relacionados con la propia salud; también con conductas que atentan contra el estatus quo normativo (por ejemplo, robo). Dado que el estudio no aborda la misma variable de interés del presente estudio, las comparaciones resultan complicadas, sin embargo, si se conceptualiza a la psicopatía como una forma de tomar riesgos innecesarios (véase Glenn et al., 2009), puede sugerirse que, en ambos estudios, IMP y FFFS son predictores importantes de la toma de decisiones riesgosas. Por lo pronto, los datos del segundo estudio sugieren que, tanto escalas relacionadas con el BAS, (IMP) y con FFFS, (DF) predicen psicopatía primaria. En el caso de la psicopatía secundaria, esta es predicha por todo el espectro de constructos que constituyen a la r-RST (BIS, BAS y FFFS). Por su parte, la conducta delincuencial solamente es predicha por sub escalas del FFFS (específicamente DF y PA). Por supuesto, las tablas 11 y 12 sugieren que el perfil RST-PQ, no siempre coincide para mujeres y hombres.

\section{Discusión General}

Vistos de manera general, ninguno de los dos estudios parece apoyar la hipótesis de Lykken (1995) en el sentido de que cada una de las psicopatías pertenece a un solo dominio de la teoría RST (o de la r-RST). En el estudio número uno, la psicopatía primaria presentó predictores tanto de BIS como de BAS. Complementariamente, la 
secundaria solamente fue predicha por BAS, sin embargo, algunas sub escalas BAS mantienen relaciones directas con la variable de interés (y otra, BAS-R mantiene una relación inversa). El segundo estudio coincide con el primero, en el sentido de que la psicopatía primaria es predicha por sub escalas relacionadas con sensibilidad al reforzamiento, y al castigo (específicamente IMP y DF). Por su parte, la secundaria es predicha por BIS, BAS (IMP) y FFFS (DF y PA). Al parecer la única variable dependiente que puede predecirse por un solo constructo de la r-RST es la conducta delincuencial, misma que es predicha por dos sub escalas del FFFS (DF y PA). El perfil diagnóstico que se observa en el primer estudio, sugiere que un individuo con diagnóstico de psicopatía primaria presentaría poca sensibilidad al castigo, y que al mismo tiempo "disfrutaría" poco de las recompensas obtenidas, (paradójicamente invierte mucho tiempo y esfuerzo en la búsqueda de dichas recompensas). Por su parte el diagnóstico de psicopatía secundaria solamente presentaría la parte paradójica del perfil (búsqueda de recompensas, pero estas producen poca gratificación). Cuando se trata de realizar un perfil diagnóstico con el RST-PQ, el individuo con diagnóstico de psicopatía primaria parecería tener problemas para controlar su comportamiento en presencia del estímulo reforzante; complementariamente poseería una creencia firme en su derecho a usar la violencia para defender sus intereses. El individuo con diagnóstico de psicopatía secundaria poseería los atributos anteriores, complementariamente, sería sensible al castigo, rápidamente se asustaría, y dicho miedo produciría respuestas autónomas que podrían ser percibidas por el individuo. Finalmente, el sujeto que presenta comportamiento delincuencial viviría en un estado de ansiedad elevado; dicho estado produciría respuestas autónomas que serían percibidas por el individuo. Adicionalmente este sujeto creería con firmeza en el uso de la violencia para defender sus intereses.

Sintetizando la información previamente expuesta los dos instrumentos de interés coinciden y difieren en cuanto a sus predicciones sobre la psicopatía primaria y secundaria. Las escalas BIS/BAS sugieren que la psicopatía primaria puede predecirse a través una combinación particular de ambas (puntajes bajos de BIS y altos de algunas escalas BAS). Por su parte, en la escala RST-PQ el BIS no hace predicción alguna de psicopatía primaria, ésta última solamente se predice por el factor de impulsividad del BAS, y el factor DF de FFFS. El resultado sugiere que los autores del RST-PQ no solamente subdividieron la RST, también modificaron de manera radical la escala BIS. La idea anterior parece confirmarse observar que al usar las escalas BIS/BAS, el sistema de inhibición conductual no es un predictor de psicopatía secundaria, pero sí lo es al usar RST-PQ. Otra inconsistencia importante entre las escalas, aparece cuando se observa la capacidad predictiva del BAS. Al usar las escalas BIS/BAS queda claro que BAS-B y BAS-M son predictores consistentes de ambas psicopatías. Al usar RST-PQ solamente un factor del BAS (IMP) hace predicciones consistentes entre las psicopatías. Así pues, de nueva cuenta queda claro que los autores de la escala en cuestión también conceptualizaron de forma, radicalmente diferente, el 
sistema de activación conductual (probablemente agruparon BAS-B y BAS-M en un solo factor, entre otras cosas). En síntesis, las escalas de interés para el presente estudio, pueden provenir de un mismo modelo científico (la TSR), sin embargo, su uso para identificar y predecir comportamiento psicopático y delincuencial no es idéntico (a veces, ni siquiera es congruente teóricamente). El desencanto generalizado de los científicos con la TSR, y las escalas asociadas, ha llevado al desarrollo de la r-TSR y a una serie de instrumentos asociados con la misma (Corr, 2016). A la fecha, la mayor parte de la investigación sobre el RST-PQ se ha centrado en determinar las propiedades psicométricas de la misma, en Inglaterra (Corr \& Cooper, 2016); en Turquía (Bahtiyar et al., 2019) y en Alemania (Dierickx et al., 2020; Pugnaghi et al., 2018). Hasta donde los autores saben, este es el primer estudio enfocado, específicamente, en comparar el RST-PQ (y a su antecesor), en cuanto a la capacidad de ambos para evaluar teorías específicas. Ambos instrumentos coinciden en rechazar la teoría de la psicopatía de Lykken. El resultado podría interpretarse en términos de que las escalas poseen validez empírica. A pesar de lo anterior, dado que los argumentos de las escalas para rechazar la teoría no siempre coinciden, (sobre todo en lo referente a la escala BIS), usar el presente estudio para justificar la validez empírica de las escalas es complicado.

De acuerdo con Corr (2010), la teoría de Likken resultaba atractiva por su parsimonia. Específicamente permitía vincular cada una de las psicopatías con una arquitectura neurofisiológica particular (psicopatía primaria con deficiencias en el sistema septo-hipocampal; la psicopatía secundaria con una hiperactividad de las vías catecolaminérgicas y dopaminérgicas). El presente estudio sugiere que el modelo puede ser parsimonioso, pero los datos empíricos no son congruentes con el mismo. Los datos de este estudio sugieren la necesidad de plantear un modelo neurofisiológico en el cual interactúen los sistemas de inhibición, activación conductual y FFFS. Finalmente, gran parte de las razones que llevaron a plantear la r-TSR tienen que ver con la ortogonalidad de los sistemas BIS y FFFS. Los datos del segundo estudio sugieren que, efectivamente, el comportamiento de estos sistemas pudiera ser independiente (ya que no siempre hacen las mismas predicciones conductuales).

Desde la adaptación de las escalas BIS/BAS a población mexicana, los autores del presente estudio han notado un interés por emplear la escala en cuestión en el país. Es importante que los profesionales de la psicología en México, tengan claro que la escala BIS/BAS ya no representa la versión más actualizada de la TSR. Complementariamente es importante que sepan que teorías clásicas de la psicopatía (como aquellas propuestas por Gray, 1970; Lykken, 1995) carecen de sustento empírico. El desarrollo de la r-RST ha dado origen a nuevos instrumentos. Como se ha demostrado en el presente estudio, los cuestionarios no siempre muestran congruencia predictiva (o siquiera conceptual). Así pues, el empleo de las teorías (o de los cuestionarios) tiene que llevarse a cabo con reservas. Adicionalmente, no debe perderse de vista que las muestras empleadas en los presentes 
estudios son de estudiantes universitarios, y que eso representa una seria limitación de los mismos (sobre todo en cuanto a su validez externa con población en situación de cárcel). Complementariamente, aún se carece de datos relacionados con la validez de constructo del RST-PQ en población mexicana. Así pues, los autores aceptan que ofrecen a los lectores trabajos preliminares. Aceptan incluso que aún es necesario profundizar sobre las teorías y los cuestionarios que se usan para predecir psicopatía y conducta delincuencial en población mexicana. La advertencia va aún más allá. Tanto dentro como fuera de México, las escalas BIS/BAS han mostrado problemas psicométricos graves (véase Barranco et al., 2009; Corr \& McNaughton, 2012). Por su parte los escasos estudios conducidos a la fecha sobre el RST-PQ arrojan datos que no muestran, incontrovertiblemente, que dicho cuestionario posea validez de constructo, invarianza de medida o consistencia interna (Pulido, 2021). Como importadores de tecnologías extranjeras, los presentes autores invitan al gremio de la psicología mexicana a ser cautelosos en el uso de estos instrumentos -y teorías-.

Quizá el pendiente más importante del presente estudio sea el de poder estudiar los datos del RST-PQ en México (y determinar si el cuestionario posee validez, confiabilidad y otras propiedades psicométricas deseables). En caso de que el instrumento resulte aceptable, otro estudio urgente sería el de evaluar la generalidad de los datos en estudiantes universitarios en población de cárcel (que es donde se presentan los casos más extremos de psicopatía y conducta delincuencial, Newman et al., 2005). Por supuesto la replicación de los resultados del presente estudio también sería deseable.

\section{Agradecimientos}

Los autores desean agradecer al APIEC-UIC, a la Universidad Anáhuac y al Centro de Estudios Superiores Monte Fénix por su apoyo para la conducción de la presente investigación. Los autores están en particular deuda con Ricardo Rivas, Ernesto Reyes, Perla Vázquez y Diana Betancourt, por su apoyo al estudio.

\section{Referencias}

Amiri, S., Babaei Nadilyu, K., \& Ghasemzade, M. (2019). Reliability, validity, and factor structure of the Persian version of the Children Reinforcement Sensitivity Scale. Iranian Journal of Psychiatry and Clinical Psychology, 24(4), 444-457. https://doi.org/10.32598/ijpcp.24.4.444

Bahtiyar, I., Corr, P. J., \& Krupić, D. (2019). The psychometric properties of the Turkish version of Reinforcement Sensitivity Theory-Personality Questionnaire-Children (RST-PQ-C). Personality and Individual Differences, 148, 73-76. https://doi.org/10.1016/j.paid.2019.05.019

Barranco, L., Rodarte, B., Medina, Y., \& Solís-Cámara, P. (2009). Evaluación psicométrica de los sistemas de activación e inhibición del comportamiento en adultos mexicanos. Anales de Psicología, 25, 358-367. 
Bijttebier, P., Beck, I., Claes, L., \& Vandereycken, W. (2009). Gray's Reinforcement Sensitivity Theory as a framework for research on personality-psychopathology associations. Clinical Psychology Review, 29(5), 421-430. https://doi.org/10.1016/j.cpr.2009.04.002

Blanchard, R. J., Blanchard, D. C., Rodgers, J., \& Weiss, S. M. (1990). The characterization and modelling of antipredator defensive behavior. Neuroscience \& Biobehavioral Reviews, 14(4), 463-472. https://doi.org/1 0.1016/S0149-7634(05)80069-7

Carver, C. S., \& White, T. L. (1994). Behavioral inhibition, behavioral activation, and affective responses to impeding reward and punishment: The BIS/BAS Scales. Journal of Personality and Social Psychology, 67(2), 319-333. https://doi.org/10.1037/0022-3514.67.2.319

Cooper, A. J., Perkins, A. M., \& Corr, P. J. (2007). A confirmatory factor analytic study of anxiety, fear, and behavioral inhibition system measures. Journal of Individual Differences, 28(4), 179-187. https://doi.org/10.1027/16140001.28.4.179

Coplan, R. J., Wilson, J., Frohlick, S. L., \& Zelenski, J. (2006). A person-oriented analysis of behavioural inhibition and behavioural activation in children. Personality and Individual Differences, 41 (5), 917-927. https://doi.org/1 0.1016/j.paid.2006.02.019

Corr, P. J. (2008). Reinforcement Sensitivity Theory (RST): Introduction. En P. J. Corr (Ed.), The reinforcement sensitivity theory of personality (pp. 143). Cambridge University Press. https://doi.org/10.1017/CBO978051 1819384.002

Corr, P. J. (2010). The psychoticism-psychopathy continuum: A neuropsychological model of core deficits. Personality and Individual Differences, 48(6), 695-703. https://doi.org/10.1016/j.paid.2009.12.023

Corr, P. J. (2016). Reinforcement sensitivity theory of personality questionnaires: Structural survey with recommendations. Personality and Individual Differences, 89, 60-64. https://doi.org/10.1016/j.paid.2015.0 9.045

Corr, P. J., \& Cooper, A. J. (2016). The Reinforcement Sensitivity Theory of Personality Questionnaire (RST-PQ): Development and validation. Psychological Assessment, 28(11), 1427- 1440. https://doi.org/10.1037/p as0000273

Corr, P. J., \& McNaughton, N. (2012). Neuroscience and approach/avoidance personality traits: A two stage (valuation-motivation) approach. Neuroscience \& Biobehavioral Reviews, 36(10), 2339-2354. https://doi.or g/10.1016/j.neubiorev.2012.09.013

Dierickx, S., Smits, D., Corr, P. J., Hasking, P., \& Claes, L. (2020). The Psychometric properties of a brief Dutch version of the Reinforcement Sensitivity Theory of Personality Questionnaire. Psychological Test Adaptation and Development, 1-11. https://doi.org/10.1027/2698-1866 /a000004

Eriksson, L. J., Jansson, B., \& Sundin, Ö. (2019). Psychometric properties of a Swedish version of the reinforcement sensitivity theory of personality questionnaire. Nordic Psychology, 71(2), 134-145. https://doi.org/10.108 $0 / 19012276.2018 .1516563$ 
Estes, W. K., \& Skinner, B. F. (1941). Some quantitative properties of anxiety. Journal of Experimental Psychology, 29(5), 390-400. https://doi.org/10.1 037/h0062283

Farrell, N., \& Walker, B. R. (2019). Reinforcement sensitivity theory and problem gambling in a general population sample. Journal of Gambling Studies, 35(4), 1163-1175. https://doi.org/10.1007/s10899-019-098503

Franken, I. H., \& Muris, P. (2005). Individual differences in decision-making. Personality and Individual Differences, 39(5), 991-998. https://doi.org/1 0.1016/j.paid.2005.04.004

Franken, I. H., Muris, P., \& Rassin, E. (2005). Psychometric properties of the Dutch BIS/BAS scales. Journal of Psychopathology and Behavioral Assessment, 27(1), 25-30. https://doi.org/10.1007/s10862-005-3262-2

Gaher, R. M., Hahn, A. M., Shishido, H., Simons, J. S., \& Gaster, S. (2015). Associations between sensitivity to punishment, sensitivity to reward, and gambling. Addictive Behaviors, 42, 180-184. https://doi.org/10.1016/j.ad dbeh.2014.11.014

Glenn, A. L., Raine, A., \& Schug, R. A. (2009). The neural correlates of moral decision-making in psychopathy. Molecular Psychiatry, 14(1), 5-6. https: //doi.org/10.1038/mp.2008.104

Gray J. A. (1981). A critique of Eysenck's Theory of Personality. En H.J. Eysenck (Ed.), A model for personality (pp. 246-276). Springer. https://doi.org/10 .1007/978-3-642-67783-0_8

Gray, J. A. (1982) The neuropsychology of anxiety: An enquiry into the functions of the septo-hippocampalsystem. Oxford University Press.

Gray, J. A. (1970). The psychophysiological basis of introversion-extraversion. BehaviourResearch and Therapy, 8(3), 249-266. https://doi.org/10.1016/ 0005-7967(70)90069-0

Gray, J. A. (1987). The psychology of fear and stress (Vol. 5). CUP Archive. Cambridge University Press.

Gray, J. A., \& McNaughton, N. (2000). The neuropsychology of anxiety: An enquiry into the functions of the septo-hippocampal system. Oxford University Press.

Gray, J. A., \& McNaughton, N. (1996). The neuropsychology of anxiety: Reprise. En D. A. Hope (Ed.), Current theory and research in motivation, Vol. 43. Nebraska Symposium on Motivation, 1995: Perspectives on anxiety, panic, and fear (pp. 61-134). University of Nebraska Press.

Hare, R. D., \& Hart, S. D. (1993). Psychopathy, mental disorder, and crime. En S. Hodgins (Ed.), Mental disorder and crime (pp. 104-115). Sage Publications.

Hughes, K. A., Moore, R. A., Morris, P. H., \& Corr, P. J. (2012). Throwing light on the dark side of personality: Reinforcement sensitivity theory and primary/secondary psychopathy in a student population. Personality and Individual Differences, 52(4), 532-536. https://doi.org/10.1016/j.paid.20 11.11 .010

Hundt, N. E., Kimbrel, N. A., Mitchell, J. T., \& Nelson-Gray, R. O. (2008). High BAS, but not low BIS, predicts externalizing symptoms in adults. Personality and Individual Differences, 44(3), 565-575. https://doi.org/1 0.1016/j.paid.2007.09.018 
Jackson, C. J. (2009). Jackson-5 scales of revised Reinforcement Sensitivity Theory (r-RST) and their application to dysfunctional real world outcomes. Journal of Research in Personality, 43(4), 556-569. https://doi .org/10.1016/j.jrp.2009.02.007

Kimbrel, N. A. (2008). A model of the development and maintenance of generalized social phobia. Clinical Psychology Review, 28(4), 592-612. htt ps://doi.org/10.1016/j.cpr.2007.08.003

Kimbrel, N. A., Nelson-Gray, R. O., \& Mitchell, J. T. (2007). Reinforcement sensitivity and maternal style as predictors of psychopathology. Personality and Individual Differences, 42(6), 1139-1149. https://doi.org/10.1016/j. paid.2006.06.028

Kramer, S., Rodriguez, B. F., \& Kertz, S. J. (2015). Predicting socially anxious group membership using reinforcement sensitivity theory. Personality and Individual Differences, 86, 474-476. https://doi.org/10.1016/j.paid.2015 .06 .053

Leone, L., \& Russo, P. M. (2009). Components of the behavioral activation system and functional impulsivity: A test of discriminant hypotheses. Journal of Research in Personality, 43(6), 1101-1104. https://doi.org/10. 1016/j.jrp.2009.08.004

Loxton, N. J., \& Tipman, R. J. (2017). Reward sensitivity and food addiction in women. Appetite, 115, 28-35. https://doi.org/10.1016/j.appet.2016.1 0.022

Luengo, M. A., Otero, J. M., Romero, E., Gómez-Fraguela, X. A., \& Tavares, E. T. (1999). Análisis de items para la evaluación de la conducta antisocial: un estudio transcultural. Revista iberoamericana de Diagnóstico y Evaluación Psicológica, 1, 21-36.

Lykken, D. T. (1995). The antisocial personalities. Lawrence Erlbaum Associates.

McNaughton, N., \& Corr, P. J. (2004). A two-dimensional neuropsychology of defense: Fear/anxiety and defensive distance. Neuroscience \& Biobehavioral Reviews, 28(3), 285-305. https://doi.org/10.1016/j.neubio rev.2004.03.005

McNaughton, N., \& Corr, P.J. (2008). The neuropsychology of fear and anxiety: A foundation for Reinforcement Sensitivity Theory. En P. J. Corr (Ed.), The reinforcement sensitivity theory of personality (pp. 44-94). Cambridge University Press. https://doi.org/10.1017/CBO9780511819384.003

Newman, J. P., MacCoon, D. G., Vaughn, L. J., \& Sadeh, N. (2005). Validating a distinction Between primary and secondary psychopathy with measures of Gray's BIS and BAS constructs. Journal of Abnormal Psychology, 114(2), 319-323. https://doi.org/10.1037/0021-843X.114.2.319

Perkins, A. M., Inchley-Mort, S. L., Pickering, A. D., Corr, P. J., \& Burgess, A. P. (2012). A facial expression for anxiety. Journal of Personality and Social Psychology, 102(5), 910-924. https://doi.org/10.1037/a0026825

Pinto-Meza, A., Caseras, X., Soler, J., Puigdemont, D., Pérez, V., \& Torrubia, R. (2006). Behavioural inhibition and behavioural activation systems in current and recovered major depression participants. Personality and Individual Differences, 40(2), 215-226. https://doi.org/10.1016/j.paid.20 05.06.021

Pugnaghi, G., Cooper, A., Ettinger, U., \& Corr, P. J. (2018). The psychometric properties of the German language reinforcement sensitivity theory- 
personality questionnaire (RST-PQ). Journal of Individual Differences, 39(3), 182-190. https://doi.org/10.1027/1614-0001/a000262

Pulido, M. (2021). The Psychometric Properties of the Spanish Language Reinforcement Sensitivity Questionnaire (RST-PQ) [Manuscrito enviado para dictamen]. Personality and Individual Differences.

Pulido, M. A., Rivera, L., Fondón, A., \& Vázquez, P. (2016a). Propiedades psicométricas de la escala BIS/BAS en una muestra de estudiantes universitarios mexicanos. Enseñanza e Investigación en Psicología, 21(3), 300-310.

Pulido, M., Hernández, A.M., Irecta, C., Reynoso, M., Silos, M. J., Valdés, D., \& Valenzuela, M. (2016b). Comorbilidad entre alcohol, tabaco y adicción a las nuevas tecnologías de la información: evaluación del modelo BIS/BAS. Revista Mexicana de Investigación en Psicología, 8(1), 19-35.

Reuter, M., Cooper, A. J., Smillie, L. D., Markett, S., \& Montag, C. (2015). A new measure for the revised reinforcement sensitivity theory: Psychometric criteria and genetic validation. Frontiers in Systems Neuroscience, 9, 38. https://doi.org/10.3389/fnsys.2015.00038

Ross, S. R., Benning, S. D., Patrick, C. J., Thompson, A., \& Thurston, A. (2009). Factors of the Psychopathic Personality Inventory: Criterionrelated validity and relationship to the BIS/BAS and Five-Factor Models of Personality. Assessment, 16(1), 71-87. https://doi.org/10.1177/10731 91108322207

Ross, S. R., Molto, J., Poy, R., Segarra, P., Pastor, M. C., \& Montanés, S. (2007). Gray's model and psychopathy: BIS but not BAS differentiates primary from secondary psychopathy in noninstitutionalized young adults. Personality and Individual Differences, 43(7), 1644-1655. https:// doi.org/10.1016/j.paid.2007.04.020

Russo, P. M., Leone, L., Lauriola, M., \& Lucidi, F. (2008). Impulsivity and reward sensitivity within the pen model: A test of discriminant hypotheses. Personality and Individual Differences, 45(7), 624-629. https: //doi.org/10.1016/j.paid.2008.07.002

Satchell, L. P., Bacon, A. M., Firth, J. L., \& Corr, P. J. (2018). Risk as reward: Reinforcement sensitivity theory and psychopathic personality perspectives on everyday risk-taking. Personality and Individual Differences, 128, 162-169. https://doi.org/10.1016/j.paid.2018.02.039

Schienle, A., Schäfer, A., Hermann, A., \& Vaitl, D. (2009). Binge-eating disorder: Reward sensitivity and brain activation to images of food. Biological Psychiatry, 65(8), 654-661. https://doi.org/10.1016/j.biopsych .2008 .09 .028

Segarra, P., Ross, S. R., Pastor, M. C., Montanés, S., Poy, R., \& Molto, J. (2007). MMPI-2 predictors of Gray's two-factor reinforcement sensitivity theory. Personality and Individual Differences, 43(3), 437-448. https://doi.org/1 0.1016/j.paid.2006.12.013

Skeem, J. L., Poythress, N., Edens, J. F., Lilienfeld, S. O., \& Cale, E. M. (2003). Psychopathic personality or personalities? Exploring potential variants of psychopathy and their implications for risk assessment. Aggression and Violent Behavior, 8(5), 513-546. https://doi.org/10.1016/S1359-1789(0 2)00098-8

Smederevac, S., Mitrović, D., Čolović, P., \& Nikolašević, Ž. (2014). Validation of the measure of revised reinforcement sensitivity theory constructs. 
Journal of Individual Differences, 35(1), 12-21. https://doi.org/10.1027/ 1614-0001/a000121

Studer, J., Baggio, S., Dupuis, M., Mohler-Kuo, M., Daeppen, J. B., \& Gmel, G. (2016). Drinking motives as mediators of the associations between reinforcement sensitivity and alcohol misuse and problems. Frontiers in Psychology, 7, 718. https://doi.org/10.3389/fpsyg.2016.00718

Torrubia, R., Avila, C., Moltó, J., \& Caseras, X. (2001). The Sensitivity to Punishment and Sensitivity to Reward Questionnaire (SPSRQ) as a measure of Gray's anxiety and impulsivity dimensions. Personality and Individual Differences, 31(6), 837-862. https://doi.org/10.1016/S0191-8 869(00)00183-5

Uzieblo, K., Verschuere, B., \& Crombez, G. (2007). The Psychopathic Personality Inventory: Construct validity of the two-factor structure. Personality and Individual Differences, 43(4), 657-667. https://doi.org/1 0.1016/j.paid.2007.01.008

Voigt, D. C., Dillard, J. P., Braddock, K. H., Anderson, J. W., Sopory, P., \& Stephenson, M. T. (2009). BIS/BAS scales and their relationship to risky health behaviours. Personality and Individual Differences, 47(2), 89-93. h ttps://doi.org/10.1016/j.paid.2009.02.003

Wardell, J. D., O'Connor, R. M., Read, J. P., \& Colder, C. R. (2011). Behavioral approach system moderates the prospective association between the behavioral inhibition system and alcohol outcomes in college students. Journal of Studies on Alcohol and Drugs, 72(6), 1028-1036. https://doi.o $\mathrm{rg} / 10.15288 /$ jsad.2011.72.1028

Wey, A., Connett, J., \& Rudser, K. (2015). Combining parametric, semiparametric, and non-parametric survival models with stacked survival models. Biostatistics, 16(3), 537-549. https://doi.org/10.1093/biostatisti $\mathrm{cs} / \mathrm{kxv} 001$ 